\title{
Parallel Transport Frame of Smarandache Curves in Euclidean Space
}

\section{Shankar Lal ${ }^{*}$}

${ }^{1}$ Department of Mathematics, H.N.B. Garhwal University, S.R.T. Campus, Tehri Garhwal, Uttarakhand, India

*Corresponding Author Email id: shankar_alm@yahoo.com

Received: 09.04.2021; Revised: 26.05.2021; Accepted: 29.05.2021

CSociety for Himalayan Action Research and Development

\begin{abstract}
In the present work, we introduce Parallel transport frames of Smarandache curves in Euclidean space. In the first section, we give the basic tools of a parallel transport frame of a curve in 4-dimensional Euclidean space. In the second section, we study Smarandache curve of Euclidean space in parallel transport frame, we solve a few theorems, corollaries and examples. Again third section, we define parallel transport frame to the Smarandache curve and obtain some definitions and their apparatus. Further fourth section, we have also explained to Frenet frame of principal normal, binomial and their derivatives in the curvatures of the curve. In the end section, we discussed about the Smarandache curve in the Euclidean space of all apparatus Frenet-Serret in the differential geometry.
\end{abstract}

2010 MSC: 14H45 14H50 53A04

Key Words: Smarandache Curves • Frenet-Serret • Euclidean Space • Parallel transport frame

\section{Introduction}

Let $\beta: I \subset R \rightarrow E^{4}$ be in $E^{4} . X=\left(x_{1}, x_{2}, x_{3}, x_{4}\right), Y=\left(y_{1}, y_{2}, y_{3}, y_{4}\right)$, and $Z=\left(z_{1}, z_{2}, z_{3}, z_{4}\right)$, where $X, Y, Z$ be any three vectors in $E^{4}$. Where $\beta$ is parameterized by arc length of $s$ if $\left\langle\beta^{\prime}(s), \beta^{\prime}(s)\right\rangle=1$, together with the inner product of $E^{4}$ given by

(1.1) $\quad<X, Y>=x_{1} y_{1}+x_{2} y_{2}+x_{3} y_{3}+x_{4} y_{4}$

$X \in E^{4}$ is

$$
\begin{aligned}
\|X\|=\sqrt{<X, X>}=\sqrt{x_{1}^{2}+x_{2}^{2}+x_{3}^{2}+x_{4}^{2}} & \Rightarrow \quad\left\|x_{4}\right\|_{\infty}=\max \left|x_{4}\right| \\
\|X\|=\left(\sum_{i=1}^{4}\left|x_{4}\right|^{p}\right)^{\frac{1}{p}}, \quad p=1 \quad & \Rightarrow \quad \frac{\partial}{\partial x}\|x\|_{p}=\frac{x \bullet\left|x_{k}\right|^{p-2}}{\|x\|_{p}^{p-1}}
\end{aligned}
$$

If $p=2$, then $\quad \frac{\partial}{\partial x}\|x\|_{2}=\frac{x}{\|x\|_{2}}$

Then their vector product is defined by the determinant 


$$
X \times Y \times Z=\left|\begin{array}{cccc}
a & b & c & d \\
x_{1} & x_{2} & x_{3} & x_{4} \\
y_{1} & y_{2} & y_{3} & y_{4} \\
z_{1} & z_{2} & z_{3} & z_{4}
\end{array}\right|
$$

Suppose $\left(t, m_{1}, m_{2}, m_{3}\right)$ be the working Frenet frame of curve $\beta$. Then $t, n, b_{1}$ and $b_{2}$ are the tangent, the principal normal, and $1^{\text {st }}$ and $2^{\text {nd }}$ binomial vectors of the curve $\beta$, respectively. Then Frenet-Serret frame is given by

$$
\left[\begin{array}{l}
t^{\prime} \\
n^{\prime} \\
b_{1}^{\prime} \\
b_{2}^{\prime}
\end{array}\right]=\left[\begin{array}{cccc}
0 & \kappa & 0 & 0 \\
-\kappa & 0 & \tau & 0 \\
0 & -\tau & 0 & \sigma \\
0 & 0 & -\sigma & 0
\end{array}\right]\left[\begin{array}{l}
t \\
n \\
b_{1} \\
b_{1}
\end{array}\right]
$$

Where

$$
\begin{aligned}
& <t, t>=<n, n>=<b_{1}, b_{1}>=<b_{2}, b_{2}>=1, \\
& <t, n>=<t, b_{1}>=<n, b_{1}>=<t, b_{2}>=<n, b_{2}>=0
\end{aligned}
$$

Here $\kappa, \tau, \sigma$ denote principal curvature to the Serret-frenet of the curve $\beta$. While $t^{\prime}, n^{\prime}, b_{1}^{\prime}$ and $b_{2}^{\prime}$ are called tangent, principal normal and 1 st and $2^{\text {nd }}$ binomial.

Let $\beta=\beta(t)$ be in $E^{4}$. The curve $\beta$ can be solved by

$$
\begin{aligned}
& t=\frac{\beta^{\prime}}{\left\|\beta^{\prime}\right\|} \\
& n=\left\|\beta^{\prime}\right\|^{2} \beta^{\prime \prime}-<\beta^{\prime}, \beta^{\prime \prime}>\beta^{\prime}, \quad b_{1}=\rho\left(b_{2} \times t \times n\right) \\
& b_{2}=\rho\left\{\frac{t \times n \times \beta^{\prime \prime \prime}}{\left\|t \times n \times \beta^{\prime \prime \prime}\right\|}\right\}, \quad \kappa=\frac{\left\|\beta^{\prime}\right\| \beta^{\prime \prime}-<\beta^{\prime}, \beta^{\prime \prime}>\|}{\left\|\beta^{\prime}\right\|^{4}} \\
& \tau=\frac{\left\|t \times n \times \beta^{\prime \prime \prime}\right\| \beta^{\prime} \|}{\|\| \beta^{\prime}\left\|^{2} \beta^{\prime \prime}-<\beta^{\prime}, \beta^{\prime \prime}>\beta^{\prime}\right\|}, \quad \sigma=\frac{<\beta^{\prime \prime \prime \prime}, b_{2}>}{\left\|t \times n \times \beta^{\prime \prime \prime}\right\|\left\|\beta^{\prime}\right\|} .
\end{aligned}
$$

Where $\rho$ is taken \pm 1 such that $\left[t, n, b_{1}, b_{2}\right]=1$.

We use $T(s)$ and vector fields $m_{1}, m_{2}$ and $m_{3}$ to construct an alternative frame. The reason for the name parallel transport frame is because the normal component of the derivatives of the field is zero. If the set $\left(t, m_{1}, m_{2}, m_{3}\right)$ as parallel transport frame and

$$
k_{1}=<t^{\prime}, m_{1}>, \quad k_{2}=<t^{\prime}, m_{2}>, \quad k_{3}=<t^{\prime}, m_{3}>
$$

Parallel transport curvatures.

Using Euler angles an arbitrary rotation matrix is given by

$$
\left[\begin{array}{ccc}
\cos \theta \cos \psi & -\cos \phi \sin \phi+\sin \phi \sin \theta \sin \psi & \sin \phi \sin \psi+\cos \phi \sin \theta \cos \psi \\
\cos \theta \cos \psi & \cos \phi \cos \psi+\sin \phi \sin \theta \sin \psi & -\sin \phi \cos \psi+\cos \phi \sin \theta \sin \psi \\
-\sin \theta & \sin \phi \cos \theta & \cos \phi \cos \theta
\end{array}\right]
$$

, Where angels $\theta, \phi, \psi$ are Euler angles. 


\section{Smarandache curve of Euclidean Space in parallel transport frame}

We define the parallel transport frame of a Smarandache curve and use Euler angles to define the relationships between the frame and the Frenet frame of the Smarandache curve in Euclidean space. For the first time in 4-dimensional Euclidean space, a well-known relation in Euclidean space is generalized.

Theorem 2.1. Let $\left(t, m_{1}, m_{2}, m_{3}\right)$ be curve $\beta: I \subset R \rightarrow E^{4}$ and $\left(t, m_{1}, m_{2}, m_{3}\right)$ denotes the curve $\beta$. The relation may be expressed as the arc is given by

$$
\begin{aligned}
& t=t(s), \\
& n=m_{1}(\cos \lambda \cos \mu)+m_{2}(-\cos \eta \sin \mu+\sin \eta \sin \lambda \cos \mu) \\
& +m_{3}(\sin \eta \sin \mu+\cos \eta \sin \lambda \cos \mu) \\
& b_{1}=m_{1}(\cos \lambda \sin \mu)+m_{2}(\cos \eta \cos \mu+\sin \lambda \sin \eta \sin \mu) \\
& +m_{3}(-\sin \eta \cos \mu+\sin \lambda \cos \eta \sin \mu) \\
& b_{2}=m_{1}(-\sin \lambda)+m_{2}(\sin \eta \cos \lambda)+m_{3}(\cos \eta \cos \lambda)
\end{aligned}
$$

Then frame $E^{4}$ are given by

$$
\left[\begin{array}{c}
t^{\prime} \\
m_{1}^{\prime} \\
m_{2}^{\prime} \\
m_{3}^{\prime}
\end{array}\right]=\left[\begin{array}{cccc}
0 & k_{1} & k_{2} & k_{3} \\
-k_{1} & 0 & 0 & 0 \\
-k_{2} & 0 & 0 & 0 \\
-k_{3} & 0 & 0 & 0
\end{array}\right]\left[\begin{array}{c}
t \\
m_{1} \\
m_{2} \\
m_{3}
\end{array}\right]
$$

where $k_{1}, k_{2}$ and $k_{3}$ are curve $\beta$ as follows

$$
\begin{array}{lr}
k_{1}=\kappa \cos \lambda \cos \eta, & k_{2}=\kappa(-\cos \eta \sin \mu+\sin \lambda \sin \eta \cos \mu), \\
k_{3}=\kappa(\sin \eta \sin \mu+\sin \lambda \cos \eta \cos \mu) & \text { and } \kappa=\sqrt{k_{1}^{2}+k_{2}^{2}+k_{3}^{2}}, \\
\tau=-\psi^{\prime}+\phi^{\prime} \sin \lambda, \quad \sigma=\frac{\lambda^{\prime}}{\sin \mu}, & \eta \cos \lambda+\lambda^{\prime} \cot \mu=0 .
\end{array}
$$

Where $\lambda^{\prime}=\frac{\sigma}{\sqrt{\kappa^{2}+\tau^{2}}}, \quad \mu^{\prime}=-\tau-\sigma \frac{\sqrt{\sigma^{2}-\lambda^{\prime 2}}}{\sqrt{\kappa^{2}+\tau^{2}}}, \quad \eta^{\prime}=-\frac{\sqrt{\sigma^{2}-\lambda^{\prime 2}}}{\cos \lambda}$.

Proof: Given that the above theorem, differentiating the $m_{1}, m_{2}, m_{3}$ with respect to $s$, we get

$$
\begin{aligned}
m_{1}^{\prime}=( & -\kappa \cos \lambda \cos \mu) t+\left(\lambda^{\prime} \sin \lambda \cos \mu-\mu^{\prime} \cos \lambda \sin \mu-\tau \cos \lambda \sin \mu\right) n \\
& +\left(\lambda^{\prime} \sin \lambda \cos \mu-\psi^{\prime} \cos \theta \sin \psi-\tau \cos \theta \sin \psi\right) n \\
& +\left(-\theta^{\prime} \sin \theta \sin \mu-\mu^{\prime} \cos \lambda \cos \mu-\sigma \sin \lambda\right) b_{1} \\
& +\left(-\lambda^{\prime} \cos \lambda-\sigma \cos \lambda \sin \mu\right) b_{2}, \\
m_{2}^{\prime}=- & \kappa[-\cos \eta \sin \mu+\sin \lambda \sin \eta \sin \mu] t+\left[\eta^{\prime} \sin \eta \sin \mu-\mu^{\prime} \cos \lambda \cos \eta\right. \\
& +\eta^{\prime} \sin \lambda \cos \eta \cos \mu+\lambda^{\prime} \sin \eta \cos \lambda \cos \mu-\mu^{\prime} \sin \lambda \sin \eta \sin -k_{2}(\cos \eta \cos \mu \\
& +\sin \lambda \sin \eta \sin \mu)] n+\left[-\lambda^{\prime} \sin \eta \cos \mu-\mu^{\prime} \cos \mu \sin \mu+\eta^{\prime} \sin \lambda \cos \eta \sin \mu\right. \\
& +\lambda^{\prime} \cos \lambda \sin \eta \sin \mu+\mu^{\prime} \sin \lambda \sin \eta \cos \mu+\tau(-\cos \eta \sin \mu+\sin \lambda \sin \eta \cos \mu)
\end{aligned}
$$




$$
\begin{aligned}
& -\sigma \cos \lambda \sin \eta] b_{1}+\left[\eta^{\prime} \cos \lambda \cos \eta-\lambda^{\prime} \sin \lambda \sin \eta\right. \\
& +\sigma(\cos \eta \sin \mu+\sin \lambda \sin \eta \sin \mu] b_{2} \\
m_{3}^{\prime}=[- & \kappa(\sin \eta \sin \mu+\sin \lambda \cos \eta \cos \mu)] t+\left[\eta^{\prime} \cos \lambda \sin \mu+\mu^{\prime} \sin \eta \cos \mu\right. \\
& -\eta^{\prime} \sin \lambda \sin \eta \cos \mu+\lambda^{\prime} \cos \lambda \cos \eta \cos \mu-\mu^{\prime} \sin \lambda \cos \eta \sin \mu-\tau(\sin \eta \cos \mu \\
& +\sin \lambda \cos \eta \sin \mu)] n+\left[\left(-\eta^{\prime} \cos \eta \cos \mu+\mu^{\prime} \sin \eta \sin \mu-\eta^{\prime} \sin \lambda \sin \eta \sin \mu\right.\right. \\
& +\lambda^{\prime} \cos \lambda \cos \eta \sin \mu+\mu^{\prime} \sin \lambda \cos \eta \cos \mu+\tau(\sin \eta \sin \mu+\sin \lambda \cos \eta \cos \mu) \\
& +\sigma(\cos \lambda \cos \eta)] b_{1}+\left(-\lambda^{\prime} \cos \lambda \sin \eta-\lambda^{\prime} \sin \lambda \cos \eta\right) \\
& +\sigma(-\sin \eta \cos \varpi+\sin \lambda \cos \eta \sin \mu)] b_{2} .
\end{aligned}
$$

Since $m_{1}, m_{2}$ and $m_{3}$ are vector field, normal component of the $m_{1}^{\prime}, m_{2}^{\prime}$ and $m_{3}^{\prime}$ must be zero and the equalities are satisfy

$$
<m_{1}^{\prime}, m_{2}>=<m_{1}^{\prime}, m_{3}>=<m_{2}^{\prime}, m_{1}>=<m_{2}^{\prime}, m_{3}>=<m_{3}^{\prime}, m_{1}>=<m_{3}^{\prime}, m_{2}>=0
$$

Also, if we consider that the parallel transport frame of the curve $\beta$ we can easily complete proof that the above theorem.

Corollary 2.1. If we consider $\lambda=\eta=\mu=0$ that then transport in $E^{4}$. We introduce the relations between the frame and the Serret-Frenet frame of a Smarandache curve in Euclidean space using Euler angles and give the parallel transport frame of a Smarandache curve. For the first time in 4-dimensional Euclidean space, the well-known Euclidean space relation is generalized.

Example 2.1. Let $\beta(s)=(\sin s, 2 s+1,2 s-1, s)$ and $\beta^{\prime \prime}(0)=(0,0,0,0)$ not calculate the Frenet at $s=0$.

$$
m_{1}=b_{1} \sin \mu, \quad m_{2}=b_{1} \cos \eta \cos \mu, \quad m_{3}=-b_{1} \sin \eta \cos \mu,
$$

Where $\eta$ and $\mu$ are constant angles.

Theorem 2.2. Suppose $\beta: I \subset R \rightarrow E^{4}$ be $k_{i}(i=1,2,3)$ in Euclidean space, then lie-down $\beta$ if $a k_{1}+b k_{2}+c k_{3}+1=0$

Proof: Let $\beta$ recline on a sphere where center is $P$ and radius is $R$, then

$$
\begin{array}{lcc}
<\beta-P, \beta-P>=R^{2} & \text { it gives us } \\
<t, \beta-P>=0, & \Rightarrow & \beta-P=\mathrm{am}_{1}+b m_{2}+c m_{3}
\end{array}
$$

For $\quad a^{\prime}=<\beta-P, m_{1}>=<t, m_{1}>+<k_{1} t, \beta-P>=0$

$<t, \beta-P>$, we get

$$
<k_{1} m_{1}+k_{2} m_{2}+k_{3} m_{3}, \beta-P>+<t, t>=a k_{1}+b k_{2}+c k_{3}+1=0 .
$$

That is, $\quad a k_{1}+b k_{2}+c k_{3}+1=0$

Moreover, $\quad R^{2}=<\beta-P, \beta-P>=a^{2}+b^{2}+c^{2}=\frac{1}{d^{2}} d$

, the plane $\quad a x+b y+c z+1=0$

Conversely, holds 


$$
a k_{1}+b k_{2}+c k_{3}+1=0, \quad P=\beta-a m_{1}-b m_{2}-c m_{3}, \quad P^{\prime}=t+\left(a k_{1}+b k_{2}+c k_{3}\right) t=0
$$

Similarly shows that $\quad R^{2}=<\beta-P, \beta-P>$ is constant.

Example 2.2. Let $\beta(s)=\left(\sin \frac{s}{\sqrt{2}}, \cos \frac{s}{\sqrt{2}}, \frac{1}{\sqrt{2}} \sin s, \frac{1}{\sqrt{2}} \cos s\right)$ be a curve in Euclidean space $E^{4}$. there are several formulas for showing that this curve is a spherical curve. But the formulas have some disadvantages which were define the above chapters. Then we calculate curvature functions of the curve $\beta$ according to parallel transport frame.

$$
k_{1}=0, k_{2}=-\cos \phi k_{3}=\sin \phi
$$

, where $\phi$ is constant. The curve $\beta$ satisfy the fallowing equation

$$
a k_{1}+b k_{2}+c k_{3}+1=0 .
$$

Consequently, the curve $\beta$ is a spherical curve. But, using the Serret-Frenet curvatures we cannot show that $\beta$ is a spherical curve. Because has a zero torsion.

\section{Parallel Transport frame in $t b_{1}$ and $t m_{1}$ to the Smarandache Curve in $E^{4}$}

$t b_{1}$ and $t m_{1}$ Smarandache Curve to parallel transport frames in $E^{4}$ then

\section{1 $t b_{1}$ Smarandache Curves with Parallel Transport Frame}

$t b_{1}$ Smarandache curves to the parallel transport frame, then obtain their Frenet apparatus.

Definition 3.1. Smarandache curve is a normal in four-dimensional Euclidean space whose location vector is determined by frame vectors.

Definition 3.2. Suppose $\beta=\beta(s)$ be curve and nonzero curvatures $k_{1}, k_{2}, k_{3}$ and $\left(t, n, b_{1}, b_{1}\right)$ be working frame on it $t b_{1}$ are $\quad \alpha\left(S_{\alpha}\right)=\frac{1}{\sqrt{2}}\left[t(s)+b_{1}(s)\right]$.

Theorem 3.1. Suppose $\beta(s)$, curvatures $k_{1}, k_{2}, k_{3}$ and $\alpha\left(s_{\alpha}\right)$ be $t b_{1}$ Smarandache curves in the frame defined by $\beta(s)$. Serret-Frenet apparatus of $\alpha\left(t_{\alpha}, n_{\alpha}, b_{1 \alpha}, b_{2 \alpha}, k_{1 \alpha}, k_{2 \alpha}, k_{3_{\alpha}}\right)$ could be of $\beta\left(t, n, b_{1}, b_{2}, k_{1}, k_{2}, k_{3}\right)$.

Proof: Let $\alpha=\alpha\left(s_{\alpha}\right)$ be $t b_{1}$ of th curve $\beta$. Then

By using (2), we get

$$
\alpha\left(S_{\alpha}\right)=\frac{1}{\sqrt{2}}\left[t(s)+b_{1}(s)\right]
$$

By differentiating (3.1)

$$
\begin{aligned}
& \frac{d \alpha\left(s_{\alpha}\right)}{d s}=\frac{d \alpha\left(s_{\alpha}\right)}{d s_{\alpha}} \cdot \frac{d s_{\alpha}}{d s}=\frac{1}{\sqrt{2}}\left[\left(k_{1}-k_{2}\right) n+k_{3} b_{2}\right], \quad \text { Then } \alpha \text { is } \\
& t_{\alpha}=A_{1} n+A_{2} b_{2}
\end{aligned}
$$


Where $\frac{d s_{\alpha}}{d s}=\sqrt{\frac{\left(k_{1}-k_{2}\right)^{2}+k_{3}^{2}}{2}}, \quad A_{1}=\frac{k_{1}-k_{2}}{\sqrt{\left(k_{1}-k_{2}\right)^{2}+k_{3}^{2}}} \quad$ and $\quad A_{2}=\frac{k_{3}}{\sqrt{\left(k_{1}-k_{2}\right)^{2}+k_{3}^{2}}}$

Again differentiating the tangent vector of the curve $\alpha$ with respect to $s_{\alpha}$, we can get $\alpha^{\prime \prime}$ as follows

$$
\alpha^{\prime \prime}=\frac{\sqrt{2}\left[-k_{1}\left(k_{1}-k_{2}\right) t+\left(k_{1} k_{2}-k_{2}^{2}-k_{3}^{2}\right) b_{1}\right]}{\left(k_{1}-k_{2}\right)^{2}+k_{3}^{2}}
$$

The principal normal of the curve $\alpha$ is

$$
n_{\alpha}=A_{3} t+A_{4} b_{1}
$$

Where $A_{3}=\frac{-k_{1}\left(k_{1}-k_{2}\right)}{\sqrt{k_{1}^{2}\left(k_{1}-k_{2}\right)^{2}+\left(k_{1} k_{2}-k_{2}^{2}-k_{3}^{2}\right)^{2}}}$ and $A_{4}=\frac{k_{1} k_{2}-k_{2}^{2}-k_{3}^{2}}{\sqrt{k_{1}^{2}\left(k_{1}-k_{2}\right)^{2}+\left(k_{1} k_{2}-k_{2}^{2}-k_{3}^{2}\right)^{2}}}$

$$
\alpha^{\prime \prime \prime}=A_{5} n+A_{6} b_{2}
$$

Where $A_{5}=\frac{2\left[-k_{1}^{2}\left(k_{1}-k_{2}\right)-k_{2}\left(k_{1} k_{2}-k_{2}^{2}-k_{3}^{2}\right)\right]}{\left[\left(k_{1}-k_{2}\right)^{2}+k_{3}^{2}\right]^{\frac{3}{2}}}$ and $A_{6}=\frac{k_{3}\left(k_{1} k_{2}-k_{2}^{2}-k_{3}^{2}\right)}{\left[\left(k_{1}-k_{2}\right)^{2}+k_{3}^{2}\right]^{\frac{3}{2}}}$

The $2^{\text {nd }} 1^{\text {st }}$ binomial vector of the curve $\alpha$ is

$$
\begin{aligned}
& b_{2 \alpha}=\frac{\left(k_{1} k_{2}-k_{2}^{2}-k_{3}^{2}\right) t+k_{1}\left(k_{1}-k_{2}\right)}{\sqrt{\left(k_{1} k_{2}-k_{2}^{2}-k_{3}^{2}\right)^{2}+k_{1}^{2}\left(k_{1}-k_{2}\right)^{2}}} \\
& b_{1 \alpha}=\frac{-k_{3} n+\left(k_{1}-k_{2}\right) b_{2}}{\sqrt{k_{3}^{2}+\left(k_{1}-k_{2}\right)^{2}}}
\end{aligned}
$$

The $1^{\text {st }}, 2^{\text {nd }}$ and $3^{\text {rd }}$ curvature of the curve $\alpha$ are

$$
\begin{aligned}
& k_{1 \alpha}=\frac{2\left[\left(k_{1} k_{2}-k_{2}^{2}-k_{3}^{2}\right)^{2}+k_{1}^{2}\left(k_{1}-k_{2}\right)^{2}\right]}{\left[k_{3}^{2}+\left(k_{1}-k_{2}\right)^{2}\right]} \\
& k_{2 \alpha}=\frac{\sqrt{2} k_{3}\left[k_{1}\left(k_{1} k_{2}-k_{2}^{2}-k_{3}^{2}\right)^{2}+k_{1}^{2}\left(k_{1}-k_{2}\right)\right]}{\left[\left(k_{3}^{2}+\left(k_{1}-k_{2}\right)^{2}\right]+\sqrt{\left(k_{1} k_{2}-k_{2}^{2}-k_{3}^{2}\right)^{2}+k_{1}^{2}\left(k_{1}-k_{2}\right)^{2}}\right.} \\
& k_{3 \alpha}=\frac{\sqrt{2}\left(-k_{1} A_{4} A_{5}-k_{2} A_{3} A_{5}-k_{3} A_{3} A_{6}\right)}{\sqrt{\left(k_{3}^{2}+\left(k_{1}-k_{2}\right)^{2}\right.}+\sqrt{\left(k_{1} k_{2}-k_{2}^{2}-k_{3}^{2}\right)^{2}+k_{1}^{2}\left(k_{1}-k_{2}\right)^{2}}}
\end{aligned}
$$

\section{4. $T M_{1}$ Smarandache curves in $E^{4}$ according to the parallel transport frame}

$T M_{1}$ Smarandache curves is curve to the parallel transport frame.

Definition 4.1. Let $\beta=\beta(s)$ be in $E^{4}$ and $\left\{T_{\beta}, M_{1 \beta}, M_{2 \beta}, M_{3 \beta}\right\}$ be its working frame. $T M_{1}$ Smarandache curves is

$$
\alpha\left(s_{\alpha}\right)=\frac{1}{\sqrt{2}}\left(T_{\beta}+M_{1 \beta}\right) .
$$


Theorem 4.1. Suppose $\beta=\beta(s)$ be curvatures $K_{1 \beta}, K_{2 \beta}, K_{3 \beta}$ and $\alpha\left(s_{\alpha}\right)$ be $T M_{1}$ in $E^{4}$ defined by $\beta=\beta(s)$. Then $\alpha$ can be $\beta$ and $\alpha\left(K_{1 \alpha}, K_{2 \alpha}, K_{3 \alpha}\right)$ can be obtained by $\beta$.

Proof: To calculate the $T M_{1}$ to $\beta(s)$ differentiating equation (4.1) with respect to $s$ then

$$
T_{\alpha} \frac{d s_{\alpha}}{d s}=\frac{1}{\sqrt{2}}\left(-K_{1 \beta} T_{\beta}+K_{1 \beta} M_{1 \beta}+K_{2 \beta} M_{2 \beta}+K_{3 \beta} M_{3 \beta}\right)
$$

The tangent vector of the curve $\beta$ can be written as

$$
T_{\alpha}=\left(\frac{-K_{1 \beta} T_{\beta}+K_{1 \beta} M_{1 \beta}+K_{2 \beta} M_{2 \beta}+K_{3 \beta} M_{3 \beta}}{\sqrt{K_{1 \beta}^{2}+K_{2 \beta}^{2}+K_{3 \beta}^{2}}}\right)
$$

Where $\frac{d s_{\alpha}}{d s}=\frac{1}{\sqrt{2}} \sqrt{K_{1 \beta}^{2}+K_{2 \beta}^{2}+K_{3 \beta}^{2}}$

Differentiating (5.3) with respect to $s$ then

$$
\begin{aligned}
& T_{\alpha}^{\prime}=\frac{d T_{\alpha}}{d s_{\alpha}}=\lambda_{0} T_{\beta}+\lambda_{1} M_{1 \beta}+\lambda_{2} M_{2 \beta}+\lambda_{3} M_{3 \beta} \\
& \text { Where } \lambda_{0}=\frac{-\sqrt{2}\left(K_{1 \beta}^{2}+K_{2 \beta}^{2}+K_{3 \beta}^{2}\right)}{\left(2 K_{1 \beta}^{2}+K_{2 \beta}^{2}+K_{3 \beta}^{2}\right)}, \quad \lambda_{1}=\frac{-\sqrt{2} K_{1 \beta}^{2}}{\left(2 K_{1 \beta}^{2}+K_{2 \beta}^{2}+K_{3 \beta}^{2}\right)} \text {, } \\
& \lambda_{2}=\frac{-\sqrt{2}\left(K_{1 \beta}^{2} K_{2 \beta}^{2}\right)}{\left(2 K_{1 \beta}^{2}+K_{2 \beta}^{2}+K_{3 \beta}^{2}\right)}, \quad \quad \lambda_{3}=\frac{-\sqrt{2}\left(K_{1 \beta}^{2} K_{3 \beta}^{2}\right)}{\left(2 K_{1 \beta}^{2}+K_{2 \beta}^{2}+K_{3 \beta}^{2}\right)}
\end{aligned}
$$

Then the curve $\alpha$ is

$$
k_{1 \alpha}=\sqrt{\lambda_{0}^{2}+\lambda_{1}^{2}+\lambda_{2}^{2}+\lambda_{2}^{3}}=\frac{\sqrt{2} \sqrt{K_{1 \beta}^{2}+K_{2 \beta}^{2}+K_{3 \beta}^{2}}}{\sqrt{2 K_{1 \beta}^{2}+K_{2 \beta}^{2}+K_{3 \beta}^{2}}}
$$

The $\alpha$ is

$$
n_{\alpha}=\frac{\lambda_{0} T_{\beta}+\lambda_{1} M_{1 \beta}+\lambda_{2} M_{2 \beta}+\lambda_{3} M_{3 \beta}}{\sqrt{\lambda_{0}^{2}+\lambda_{1}^{2}+\lambda_{2}^{2}+\lambda_{3}^{2}}}
$$

The $3^{\text {rd }}$ derivative of $\alpha$ is

$$
\begin{aligned}
& \alpha^{\prime \prime \prime}=\left(\lambda_{0} T_{\beta}^{\prime}+\lambda_{1} M_{1 \beta}^{\prime}+\lambda_{2} M_{2 \beta}^{\prime}+\lambda_{3} M_{3 \beta}^{\prime}\right) \frac{\sqrt{2}}{\sqrt{2 K_{1 \beta}^{2}+K_{2 \beta}^{2}+K_{3 \beta}^{2}}} \\
& \Rightarrow T_{\alpha} \times n_{\alpha} \times \alpha^{\prime \prime \prime}=C_{1} M_{1 \beta}+C_{2} M_{2 \beta}+C_{3} M_{3 \beta}
\end{aligned}
$$

Where $C_{1}=\frac{\sqrt{2}\left[\lambda_{0} \lambda_{3} K_{1 \beta} K_{2 \beta}-\lambda_{0} \lambda_{2} K_{1 \beta} K_{3 \beta}-\left(\lambda_{1} K_{1 \beta}+\lambda_{2} K_{2 \beta}+\lambda_{3} K_{3 \beta}\right)\left(\lambda_{3} K_{2 \beta}-\lambda_{2} K_{3 \phi}\right)\right]}{\sqrt{\lambda_{0}^{2}+\lambda_{1}^{2}+\lambda_{2}^{2}+\lambda_{3}^{2}}\left(2 K_{1 \beta}^{2}+K_{2 \beta}^{2}+K_{3 \phi}^{2}\right)}$

$$
C_{2}=\frac{\sqrt{2}\left[\lambda_{0} \lambda_{3} K_{1 \beta}^{2}-\lambda_{0} \lambda_{1} K_{1 \beta} K_{3 \beta}-\left(\lambda_{1} K_{1 \beta}+\lambda_{2} K_{2 \beta}+\lambda_{3} K_{3 \beta}\right)\left(\lambda_{3} K_{1 \beta}-\lambda_{1} K_{3 \beta}\right)\right]}{\sqrt{\lambda_{0}^{2}+\lambda_{1}^{2}+\lambda_{2}^{2}+\lambda_{3}^{2}}\left(2 K_{1}^{2}+K_{2}^{2}+K_{3}^{2}\right)}
$$




$$
C_{3}=\frac{\sqrt{2}\left[\lambda_{0} \lambda_{2} K_{1 \beta}^{2}-\lambda_{0} \lambda_{1} K_{1 \beta} K_{2 \beta}-\left(\lambda_{1} K_{1 \beta}+\lambda_{2} K_{2 \beta}+\lambda_{3} K_{3 \beta}\right)\left(\lambda_{1}-\lambda_{2}\right) K_{2 \beta}\right]}{\sqrt{\lambda_{0}^{2}+\lambda_{1}^{2}+\lambda_{2}^{2}+\lambda_{3}^{2}}\left(2 K_{1}^{2}+K_{2}^{2}+K_{3}^{2}\right)}
$$

We know the $2^{\text {nd }}$ and $1^{\text {st }}$ binomial of the curve $\alpha$ is

$$
\begin{aligned}
& b_{2 \alpha}=\frac{C_{1} M_{1 \beta}+C_{2} M_{2 \beta}+C_{3} M_{3 \beta}}{\sqrt{C_{1}^{2}+C_{2}^{2}+C_{3}^{2}}} \\
& b_{1 \alpha}=b_{2 \alpha} \times T_{\alpha} \times n_{\alpha}=\gamma_{0} T_{\beta}+\gamma_{1} M_{1 \beta}+\gamma_{2} M_{2 \beta}+\gamma_{3} M_{3 \beta}
\end{aligned}
$$

Where $\gamma_{0}, \gamma_{1}, \gamma_{2}, \gamma_{3}$ are constants and $M_{1 \alpha}, M_{2 \alpha}$, and $M_{3 \alpha}$ are frame of $M_{1 \beta}, M_{2 \beta}$, and $M_{3 \beta}$ of the curve $\alpha$ is given. Further $2^{\text {nd }}$ and $3^{\text {rd }}$ curvature tensor of the curve $\alpha$ is

$$
\begin{aligned}
& k_{2 \alpha}=\sqrt{\frac{C_{1}^{2}+C_{2}^{2}+C_{3}^{2}}{\sqrt{\lambda_{0}^{1}+\lambda_{1}^{2}+\lambda_{2}^{2}+\lambda_{3}^{2}}}} \\
& k_{3 \alpha}=\frac{-\left(C_{1} K_{1 \beta}+C_{2} K_{2 \beta}+C_{3} K_{3 \beta}\right)\left(\lambda_{1} K_{1 \beta}+\lambda_{2} K_{2 \beta}+\lambda_{3} K_{3 \beta}\right)}{\sqrt{C_{1}^{2}+C_{2}^{2}+C_{3}^{2}}}
\end{aligned}
$$

Then $1^{\text {st }}, 2^{\text {nd }}$ and $3^{\text {rd }}$ curvature of the curve $\alpha$ is

$$
\begin{aligned}
& K_{1 \alpha}=\sqrt{\lambda_{0}^{2}+\lambda_{1}^{2}+\lambda_{2}^{2}+\lambda_{3}^{2}} \cos \theta_{\alpha} \cos \psi_{\alpha} \\
& K_{2 \alpha}=\sqrt{\lambda_{0}^{2}+\lambda_{1}^{2}+\lambda_{2}^{2}+\lambda_{3}^{2}}\left[-\cos \phi_{\alpha} \sin \psi_{\alpha}+\sin \phi_{\alpha} \sin \theta_{\alpha} \cos \psi_{\alpha}\right] \\
& K_{3 \alpha}=\sqrt{\lambda_{0}^{2}+\lambda_{1}^{2}+\lambda_{2}^{2}+\lambda_{3}^{2}}\left[\sin \phi_{\alpha} \sin \psi_{\alpha}+\cos \phi_{\alpha} \sin \theta_{\alpha} \cos \psi_{\alpha}\right]
\end{aligned}
$$

\section{Discussion}

We discussed an Einstein's theory opened a door to new geometries such as Smarandache curve of parallel transport frame in the Euclidean space. They adapted the geometrical models to relativistic motion of charged particles. The dynamics of charged particles are currently defined by the Frenet-Serret formalism of relativistic motion. On another usual curve, a Smarandache is a position vector made up of Frenet frame vectors. In this paper, we investigate a unique Curve in Euclidean space.

\section{References}

Ali A T (2010) Special Smarandache curves in the Euclidean space. Int. J. Math. Combin. 2: 30-36.
Bharathi K and Nagaraj M (1985) Quaternion valued function of a real Serret- Ferret formulae. Indian J. pure appl. Math., 16 : 741-756.

Bishop L R (1975) There is more than one way to frame a curve. Amer. Math.: 82 (3): 246-251.

Celik F, Bozkurt Z, Gok I, Ekmekci F N and Yayl $Y$ (2014) Parallel transport frame in 4dimensional Euclidean space $E^{4}$.Caspian $J$. Math. Sci. (CJMS), 3(1): 91-103.

Cetin M and Kocayigit H (2013) On the quaternion Smarandache Curves in Euclidean 3-space. Int. J. Contem. Math. Sciences, 8 (no.3): 139-150.

Elzawy M (2017) Smarandache curves in Euclidean 4- space $E^{4}$. J. of Egyptian Mathematical society, 25: 268-271. 
Elzawy M and Mosa S (2017) Smarandachecurves in the Galilean 4-space $G_{4}$.J. Egypt. Math. Soc., 25: 53-56.

Krpinar T and Turhan E (2013) Biharmonic curves according to parallel transport frame in $E^{4}$.Bol. Soc. Paran. Mat. (3s), 31(2): 213-217.

Llarslan E (2008) Nesovic, Some characterizations of osculating curves in the Euclidean space. Demonstratio Mathematica, XLI (4): 931-939.

Senyurt S and Calskan A (2015) An application according to spatial quaternion Smarandache curve. Appl. Math. Sci. 9 (5): 219-228.

Turgut M and Yilmaz S (2008) Smarandache curve in Minkowaki space-time. Int. J. math. Comb. 3: 51-55.

Yilmz S and Turgut M (2010) A new version of Bishop Frame and an application to spherical image. J. Math. Anal. Appl., 371:764-776. 